Meta

Journal des tradlucteurs

Translators' Journal

\title{
Traitement des unités lexicales
}

\section{Claude Tatilon}

Volume 27, numéro 2, juin 1982

URI : https://id.erudit.org/iderudit/004324ar

DOI : https://doi.org/10.7202/004324ar

Aller au sommaire du numéro

Éditeur(s)

Les Presses de l'Université de Montréal

ISSN

0026-0452 (imprimé)

1492-1421 (numérique)

Découvrir la revue

Citer cet article

Tatilon, C. (1982). Traitement des unités lexicales. Meta, 27(2), 167-172.

https://doi.org/10.7202/004324ar

Ce document est protégé par la loi sur le droit d'auteur. L’utilisation des services d'Érudit (y compris la reproduction) est assujettie à sa politique d'utilisation que vous pouvez consulter en ligne.

https://apropos.erudit.org/fr/usagers/politique-dutilisation/
Cet article est diffusé et préservé par Érudit.

Érudit est un consortium interuniversitaire sans but lucratif composé de l'Université de Montréal, l'Université Laval et l'Université du Québec à Montréal. Il a pour mission la promotion et la valorisation de la recherche. https://www.erudit.org/fr/ 


\section{Traitement des unités lexicales*}

Claude Tatilon

La traduction étant avant tout, comme l'a dit plaisamment Valery Larbaud, une «transfusion du sens », il me semble opportun d'attirer l'attention sur les unités linguistiques qui participent le plus étroitement à la signification d'un texte, à savoir les unités lexicales. Mon propos sera donc, d'abord, de décrire ces unités pour ensuite envisager leur traitement dans la perspective d'une pratique de la traduction.

\section{LES TYPES D'UNITÉS LEXICALES: UNITÉS SIMPLES/UNITÉS ARTICULÉES}

A première vue, il peut paraître aisé au traducteur de distinguer des unités lexicales simples (ou lexèmes dans la terminologie du linguiste André Martinet) formées d'un seul mot graphique et des unités articulées (ou synthèmes chez le même) formées de deux ou plusieurs mots. (Ces dernières n'en sont pas moins des unités en vertu de leur comportement syntaxique qui est identique à celui d'une unité simple: par exemple, pomme de terre pouvant commuter dans un énoncé avec patate ou légume.)

Mais la réalité est plus complexe, précisément au niveau des unités articulées, dont il faut soigneusement distinguer trois types:

- Des synthèmes à articulation visible :

Ex.: couvre-chef, ouvre-porte, tourne-vis ... à brûle-pourpoint, à feu doux, à cor et à cri ... prendre part, prendre parti, prendre froid.

- Des synthèmes à articulation cachée, se subdivisant en:

- synthèmes monographes (formés d'un lexème libérable et d'un affixe) :

Ex.: poirier, épicier, tendrement, brunette, confrère ... ainsi que compagnon (dont la dérivation n'est plus évidente).

- syntagmes lexicalisés (dont tous les éléments sont figés et qui fonctionnent en bloc comme des unités, c.-à-d. qui peuvent commuter, dans des énoncés, avec des lexèmes synonymes) :

Ex.: jeune fille (célibataire ou vierge), sucrer les fraises (trembler), casser les pieds (ennuyer), prendre la mouche (s'emporter), mettre sur pied (organiser).

Il me paraît superflu d'insister, devant des traducteurs, sur les erreurs d'interprétation auxquelles peut donner lieu l'identification de telles unités. Mais, en

* Colloque de Glendon, 1980. 
revanche, il faut encore mentionner une réalité lexicale, située à la frontière du syntaxique, qui, elle aussi, est de nature à nous fourvoyer: les quasi-unités que sont les «expressions idiomatiques». La cohésion de leurs éléments, si elle n'est pas absolue, est néanmoins régie par des contraintes combinatoires strictes dont la transgression porte gravement atteinte à l'authenticité des expressions. Je prendrai pour exemple quelques anglicismes très répandus à Toronto: «demander" une question, "écrire" une dissertation, "appliquer» le frein, ou encore «contribuer 10\$» (au lieu de faire une contribution de ... ou, plus économique linguistiquement, donner $10 \$$ pour telle cause) et enfin une population de 30000 (habitants étant obligatoire en français malgré sa redondance avec population).

\section{L'INFORMATION LEXICALE : INFORMATION SÉMANTIQUE/ INFORMATION SOCIOLINGUISTIQUE}

L'information transmise par les unités lexicales est d'une double nature, sémantique (référentielle, cognitive) et sociolinguistique (connotative, stylistique).

\section{L'information sémantique}

Considérées à l'intérieur du répertoire de la langue, les unités lexicales possèdent un sémantisme, qui est l'ensemble des signifiés admis par chacun de leurs signifiants. C'est dire que ces unités sont en général largement polysémiques (caractère contre lequel les terminologies spécialisées s'efforcent de lutter par des moyens souvent très onéreux. Exemple: prise au domaine de la navigation maritime, cette création néologique accompagnée d'un réinvestissement encombrant de termes courants: aéroglisseur à jupe souple /aéroglisseur à jupe rigide).

Pour illustrer la polysémie lexicale, phénomène bien connu de tout utilisateur de dictionnaires, un seul exemple nous suffira:

Soit le lexème table. Sémantisme:

1. Meuble, objet utilitaire formé d'une surface plane horizontale supportée par un ou plusieurs pieds. Suit alors dans les dictionnaires, pour caractériser les différents types de tables, une importante extension synthématique, dont on peut retenir : table à dessin, - de jardin, - de jeu, - de nuit, - d'opération, - de ping-pong, - de toilette.

2. Nourriture ( $C$ ' est une des meilleures tables du pays » ou « table d'hôte»).

3. Lieu où sont pris les repas («A table!»).

4. Signifiés métaphoriques: table ronde, _ rase, _ des matières, se mettre $\grave{a}$ -

Le traducteur attentif n'aura aucun mal à constater que dans la mise en mots de son TD (texte de départ) la polysémie potentielle d'une unité est généralement annulée (sauf en cas de jeux de mots), ou pour le moins réduite, par le contexte verbal qui sélectionne le signifié adéquat, ou les signifiés admis, en oblitérant tous les autres. Ainsi: $j^{\prime}$ ai acheté une - (table), contexte très peu contraignant, admet toutes les possibilités présentées précédemment en 1. (et entre lesquelles la situation devra trancher) mais exclut celles des 2 ., 3. et 4. Un autre contexte, plus contraignant que le premier, $j$ 'ai acheté une - pour notre chambre à coucher, réduira les possibilités à deux: - de nuit, - de toilette (sauf situation 
exceptionnelle, qui imposerait par exemple une table de jardin, ou d'opération, dans une chambre!).

La situation de communication, en effet, peut à son tour jouer un rôle important. Ainsi, accompagnant une invitation à dîner, la précision : «Nous passerons à table vers 20 heures" pourrait très bien vouloir dire : «S'il vous plait, ne vous ramenez pas à 19 heures!» ou encore: "Tâchez d'être à l'heure ce coup-ci!»

Cela explique pourquoi, devant le fréquent vide situationnel du TD, le traducteur éprouve très souvent un impérieux besoin de se documenter, et pourquoi la documentation, étant une nécessité de la traduction professionnelle, est devenue un domaine à part entière de sa pédagogie : comme le prouve à l'évidence - et par l'absurde - cette perle recueillie sur l'étiquette d'un sous-vêtement de provenance américaine qui portait (en deux langues à l'intention du marché canadien) ces laconiques consignes de lavage (à la machine) : «Gentle cycle»... «Aimable bicyclette»!

\section{L'information sociolinguistique}

En traversant l'espace, le temps et les idéologies qui les habitent, les unités lexicales se chargent de sens, plus précisément de nuances sémantiques qui viennent s'ajouter à leurs dénotations (qui changent, elles aussi, qui «glissent» au cours de l'histoire des termes). Ce sont ces nuances que, sous le nom d'indices, je vais tenter de récapituler par la liste que voici :

$1^{\circ}$ Indices diachroniques (état de langue)

Ex.: des lexèmes «sucrés par l'âge » comme derechef, nonobstant, le synthème faire florès; d'autres, résolument modernes, comme cinérama, urger, marketing ou prendre son pied.

$2^{\circ} \quad$ Indices géographiques (origine géographique)

Ex.: peuchère, espaloufi, pomper l'air à quelqu'un (Provence); magané, avoir son voyage, prendre une débarque (Québec).

$3^{\circ}$ Indices sociologiques (appartenance sociale)

Ex.: les parlers de groupes non professionnels - cercles sportifs, militaires, partis politiques, communautés scolaires...

$4^{\circ} \quad$ Indices professionnels (appartenance professionnelle)

Ex.: termes techniques, tels ceux-ci, empruntés à la terminologie du matériel roulant ferroviaire français et désignant, non sans humour, les différents types de locomotives d'entretien : dameuse, régaleuse, bourreuse, cribleuse, dégarnisseuse.

$5^{\circ} \quad$ Indices artistiques (usage artistique du langage)

a) Vocabulaires "poétiques» (fossilisés dans des codes culturels: ris/ pleurs, ondelflots, nues/nuées, cavale, hymen ...)

b) Créations lexicales (celles de la littérature, de l'humour, de la publicité: «patrouillotisme» (Rimbaud) et les centaines de «mots-valises» en vogue aujourd'hui).

$6^{\circ}$ Indices de civilité (différenciation situationnelle)

Indices à quatre degrés, affectation, mondanité, familiarité, vulgarité (prendre congé, se retirer, se barrer, se tailler, foutre le camp ...) 
$7^{\circ}$ Indices d'appréciation (jugements de valeur)

Termes mélioratifs ou péjoratifs (nègre opposé à noir, marâtre à mère, garnement ou chérubin à enfant, rata à soupe, nectar à boisson ...)

En langue, s'il semble exister des unités sans indices sociolinguistiques (table, oiseau), la plupart en possèdent un ou deux, voire trois. Placée dans un texte, toute unité est susceptible de se charger d'indices supplémentaires, tirés du contexte. Ainsi, dans ce vers de Baudelaire :

Et tes pieds s'endormaient dans mes mains fraternelles
(Le Balcon)

le lexème pied, pourtant souvent affecté en langue d'un indice de familiarité voire d'appréciation péjorative (qu'on songe aux nombreuses expressions auxquelles il donne lieu), évoquant ici par métonymie la femme aimée, cristallise toute la tendresse et la sensualité qui sous-tendent l'énoncé. S'il fallait traduire ce vers dans une langue possédant comme le français plusieurs unités lexicales pour désigner le pied, partie du corps humain, on pourrait certes choisir, comme ici, le terme le plus neutre, qui se sémantiserait de lui-même au contact de son contexte verbal; ou, si cela était nécessaire (disons, pour des raisons de métrique), choisir un terme mélioratif équivalant à notre petons (souvenons-nous de ceux de Valentine), qui s'harmoniserait parfaitement avec le contexte. Mais, en aucun cas, il ne faudrait choisir un équivalent de panards ou d'arpions, car cela reviendrait, stylistiquement, à les mettre dans le plat.

\section{L'ORGANISATION DES LEXIQUES : POLYMORPHIE ET POLYSEMIE}

La conception naïve des lexiques - «sacs de mots », très préjudiciable à la pratique de la traduction, doit être énergiquement dénoncée. Dans les langues naturelles, où l'on ne constate pas d'isomorphisme lexical étendu, il est plus avisé de tenir les différences pour la règle et les ressemblances pour l'exception. Il en résulte, évidemment, que l'hypothèse d'une correspondance entre les lexiques de langues différentes est à rejeter. Voici quelques exemples empruntés à l'anglais et au français :

$1^{\circ}$ Exemple de polymorphie: le suffixe nominal français -ement, qui, sans changer le signifié du lexème (dénotant un procès), transforme un verbe en substantif :

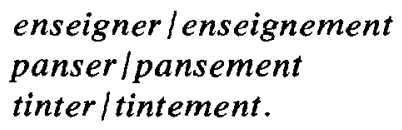

Mais, d'une part, ce modèle de formation n'est pas systématiquement utilisable (il ne fonctionne pas avec des bases comme penser, marcher, courir) et il est, d'autre part, concurrencé par d'autres modèles :

$$
\begin{array}{ccc}
\text { base verbale } & +\varnothing \text { (marche, danse, course) } \\
,, & ,, & +- \text { ation (formation, condamnation, variation) } \\
,, & , & + \text { - age (mariage, vidage, raturage) } \\
,, & \text {, } & + \text {-ure (peinture, rature, friture). }
\end{array}
$$

Il faut aussi noter qu'il existe des verbes sans substantifs correspondants : fumer, par exemple, car fumure ne dénote pas un procès mais un matériau. 
$2^{\circ}$ Exemple de polysémie : le suffixe nominal français -ure, qui, dans peinture, signifie à la fois le procès, le résultat, le matériau, la technique.

$3^{\circ}$ Exemple d'irrégularité combinatoire: en anglais, on peut parler de roast meat, de roast beef, de roast pork, même de roasted nuts, mais on doit dire baked ham, sans qu'aucune raison référentielle justifie le changement (la cuisson s'effectuant dans tous les cas à l'aide d'un four).

$4^{\circ}$ Exemples de non correspondance entre lexiques :

a) Au couple anglais deep/shallow ne correspond en français que le terme profond; inversement, à songe/ rêve ne correspond que dream.

b) Reine et queen partagent à peu près les mêmes acceptions («souveraine», «insecte», «personne très en vue dans une certaine sphère», «pièce du jeu d'échec»), mais aux cartes le français ne dit plus aujourd'hui que dame, là où l'anglais dit toujours queen.

c) Considérons enfin ce sous-ensemble :

avant-hier/ hier/ aujourd'huil demain/ après-demain

et cet autre, obtenu par translation du point de référence :

l'avant-veille/la veille/ ce jour-là/le lendemain/le surlendemain

$\mathrm{Au}$ premier correspond en anglais un ensemble à trois termes seulement : yesterday/today/ tomorrow, les extrémités n'étant plus lexicalisées et utilisant des syntagmes: - the day before yesterday/ - the day after tomorrow.

$\mathrm{Au}$ second ensemble ne correspond plus que le terme pivot, that day, les autres signifiés étant de nouveau confiés aux syntagmes - day(s) beforel - day(s) after.

Je conclurai en disant quelques mots du principe d'équivalence fonctionnelle, qui devrait, à mon sens (et à celui de nombreux théoriciens), présider à toute démarche traductionnelle. Ce principe peut s'énoncer ainsi : traduire, ce n'est pas imiter servilement les structures d'un texte de départ, c'est chercher à créer - au prix souvent de substitutions structurales plus ou moins faciles à exécuter (compensations) - un texte d'arrivée qui remplisse les mêmes fonctions que le texte de départ, c.-à-d. un TA qui soit capable de produire sur ses lecteurs des effets comparables à ceux produits sur les siens par le TD. En d'autres termes, pour nous en tenir aux unités lexicales, objet de cet exposé : un TA qui transmette la même information que le TD. Car il s'agit bien de traduire l'information, toute 1 'information - sémantique et sociolinguistique - et non les structures porteuses de cette information. Ainsi, pineapple sera évidemment traduit par ananas (non par pomme de pin) et cop parfic ou poulet (non seulement par agent de police ou policier). Toute l'information, la même information, autant que faire se peut: c'est le principe directeur. J'en donnerai deux exemples en terminant.

Le premier, à ne pas suivre, je l'ai rencontré dans une encyclopédie pour la jeunesse éditée aux États-Unis et traduite en français pour le marché québécois : à l'article Synonyme, la définition était accompagnée de cet exemple, bien peu éclairant : petit-faible! L'article américain, auquel j'eus recours pour comprendre, me donna la clé de l'énigme : son exemple était : small-little. L'explication était alors évidente : le traducteur n'avait pas vu que l'information pertinente de son TD ne résidait pas dans le sens dénotatif de chacun des deux lexèmes mais, 
bien sûr, dans leur rapport de synonymie. Il n'avait donc pas vu que la lacune du lexique français vis-à-vis de cette paire pouvait être comblée par n'importe quelle autre paire de synonymes existant en français, voiture-automobile, bateaunavire, arachide-cacahuète, aussi bien que idiot-imbécile (la synonymie, telle que la définissait l'article, se situant au seul plan de la dénotation).

La pseudo-«fidélité » de ce premier exemple est donc non pertinente. Considérons maintenant $l^{\prime}$ «infidélité » du second. À un slogan publicitaire français évoquant en des termes particulièrement heureux un parapluie télescopique :

Il vous plaira avant même qu'il ait plu

je proposerai comme équivalent cette version anglaise :

When it rains it reigns,

qui parvient, je pense, à restituer toute l'information pertinente, à savoir : le message laudatif (plaire — reign) et le message ludique, ici si important (jeux de mots construits de la même manière, sur l'homophonie de deux verbes différents). En définitive, même efficacité, mêmes vertus publicitaires des deux énoncés.

Ces deux derniers exemples vous semblent peut-être avoir perdu de vue le propos initial de mon exposé : le traitement des unités lexicales. Ils le placent, au contraire, dans la bonne perspective. Tout texte diffusé à l'intention d'un public (et les vraies traductions sont faites pour l'être) constitue un acte de communication authentique, dont la finalité est précisément l'information diversifiée qu'il transmet à ce public. Un texte traduit n'échappe pas à cette règle : les unités lexicales qu'il renferme - au même titre, du reste, que les autres structures linguistiques qui composent son être matériel — ne sont que des moyens au service de sa fin communicative. Et c'est précisément cette fin communicative qui, selon moi, devrait constituer le point de mire de tout traducteur en action. 